\title{
The role of the Ex-PRESS device implantation in the management of pseudophakic glaucoma
}

\author{
Hany Mahmoud*, Ahmed Gad Kamel**Usama Ali Mohammed***, \\ Ali Mahmoud Ismail***** \\ *Assistant lecturer of Ophthalmology, Sohag Faculty of Medicine, Sohag \\ university **lecturer of Ophthalmology, Sohag Faculty of Medicine, \\ Sohag university ***associate professor of Ophthalmology, Sohag Faculty \\ of Medicine, Sohag university**** Sohag Faculty of Medicine, Sohag \\ university.
}

\begin{abstract}
:
Purpose: evaluation of the role of the Ex-PRESS device implantation in the management of pseudophakic glaucoma.

Design: non-comparative case series study.

Patients and methods: 6 eyes with pseudophakic glaucoma scheduled to undergo Ex-PRESS glaucoma filtration device. Each study patient underwent a complete ophthalmic examination preoperatively including full clinical history taking, VA measurement, autorefraction examination, slit lamp biomicroscopy of the anterior segment, IOP measurement using Goldmann applanation tonometer, gonioscopic examination to evaluate of the angle of AC, and ophthalmoscopy of the posterior segment using a direct ophthalmoscopy. All the patients were subjected to functional and structural evaluation by SAP (HFA 745i) for central 30-2 threshold test, endothelial cell evaluation by non-contact specular microscopy (Topcon specular microscope; SP-1P Version 1.20) for endothelial cell count and OCT scans (Topcon 3D OCT-2000) for peripapillary RNFL thickness measurements, ONH analysis and GCA. All patients included were having pseudophakic OAG. Patients were excluded if they were under 18 years old. All eyes underwent Ex-PRESS device implantation performed by the same surgeon for consistency, using the standardized technique for the procedure. A complete ophthalmologic follow-up examination included IOP measurement using Goldmann applanation tonometry, number of drugs required to attain IOP control and any associated complications was carried out postoperatively at the 1st and 3rd days, the end of 1st week, every month till the end of the 6th month and the end of 1st year. Best-corrected visual acuity (BCVA) was tested using Snellen chart, which was converted to LogMAR for statistical analysis. Criteria for success were defined as follows; Absolute success: IOP $\leq 21 \mathrm{mmHg}$ without any medication, qualified success: IOP $\leq 21 \mathrm{mmHg}$ with ocular hypotensive medications. VF test with SAP, specular microscopy and ONH analysis and GCA assessed with OCT were carried out one year postoperatively.

Results: There were 4 eyes $(80 \%)$ showed complete success (IOP $<21 \mathrm{mmHg}$ without treatment) and 1 eyes (20\%) showed qualified success (IOP <21 mmHg with anti-glaucoma medical treatment). There is a statistically significant reduction in IOP 1, 3, 6 months and one year postoperatively $(P<0.001)$ with the most reduction in IOP observed at 6 months after surgery due to controlling all cases with IOP $>21 \mathrm{mmHg}$ using anti-glaucoma medications by this time. There is a statistically significant improvement of postoperative BCVA $(P=0.002)$. Moreover, there is a statistically significant reduction of number of anti-glaucoma medications used postoperatively $(P<0.001)$. For VF indices, there is a statistically significant improvement in both MD and PSD postoperatively (MD; $P<0.001$ ) (PSD; $P=0.002$ ). However; There is no significant improvement in the staging of glaucoma according to GSS2 $(P=327)$. For OCT parameter, there is a statistically significant increase in the thickness of both RNFL and GCC $(P<0.001)$. Furthermore, endothelial CD shows a statistically significant increase postoperatively $(P<0.001)$.
\end{abstract}

Conclusion: Ex-PRESS is preferable for pseudphakic glaucoma. 


\section{Introduction:}

Glaucoma in pseudophakic eyes is not uncommon. Pseudophakia by itself does not cause ocular hypertension. Pseudophakic glaucoma should be defined as glaucoma that would not be present in an eye if it were not pseudophakic. $^{(1)}$ Clear corneal phacoemulsification (CCP) is associated with significant and sustained reduction in IOP in both normal subjects and patients with POAG. ${ }^{(2-3)}$ However; The implantation of an intraocular lens (IOL) after phacoemulsification may determine the appearance of some additional mechanisms for secondary glaucoma such as pupillary block, inflammation, haemorrhage, and pigment dispersion. ${ }^{(4)}$ The pseudophakic glaucoma may occur immediately after the implantation or later during the postoperative course, including both the $\mathrm{OAG}$ and ACG.

One of the surgical treatments for pseudohakic glaucoma is glaucoma drainage device (GDD) implantation such as Ex-PRESS device. ${ }^{(5)}$ The ExPRESS miniature glaucoma implant (Alcon Laboratories Inc., Fort Worth, TX) is a biocompatible, non-valved stainless steel tube. The Ex-PRESS is currently implanted under a partial thickness sclera flap, as first suggested by Dahan and Carmicheal. ${ }^{(6)}$ the procedure is similar to standard trabeculectomy, and includes creation of scleral flap and a conjunctival filtration bleb, but no peripheral iridectomy is required.

Herein, we evaluate the role of the ExPRESS device implantation in the management of pseudophakic glaucoma.

\section{Patients and methods:}

This is a non-comparative case series study of 6 eyes with pseudophakic glaucoma that were scheduled to undergo Ex-PRESS glaucoma filtration device. All patients' data were collected between November 2016 and July 2018(the patients are operated between November 2016 to April 2017 and then followed up for one year) at Ophthalmology department - Sohag University hospital. The research adhered to the tenets of the Declaration of Helsinki. Written informed consent was taken from each patient. Each study patient underwent a complete ophthalmic examination preoperatively including full clinical history taking, VA measurement, autorefraction examination, slit lamp biomicroscopy of the anterior segment, IOP measurement using Goldmann applanation tonometer, gonioscopic examination to evaluate of the angle of $\mathrm{AC}$, and ophthalmoscopy of the posterior segment using a direct ophthalmoscopy. All the patients were subjected to functional and structural evaluation by SAP (HFA 745i) for central 30-2 threshold test, endothelial cell evaluation by non-contact specular microscopy (Topcon specular microscope; SP-1P Version 1.20) for endothelial cell count and OCT scans (Topcon 3D OCT-2000) for peripapillary RNFL thickness measurements, $\mathrm{ONH}$ analysis and GCA. All patients included were having pseudophakic OAG. Patients were excluded if they were under 18 years old.

Surgical technique: All eyes underwent Ex-PRESS device implantation performed by the same surgeon for consistency, using the standardized technique for the procedure. All the procedures were performed in the superior conjunctival area. After retrobulbar local anesthesia, superior rectus bridle suture was taken. A fornix-based conjunctival flap was created with a relaxing incision on one side. Next, a 50\% thickness rectangular scleral flap $(5 \times 5 \mathrm{~mm})$ was constructed and advanced anteriorly into the clear 
cornea using Alcon crescent knife (Saint Crescent, angled, bevel up). Paracentesis was done temporally using a 20G micro- vitreoretinal (MVR) blade. As the scleral flap was lifted, care was taken to identify the center of the "blue line" adjacent to the clear cornea, which corresponds to the location of the TM.

A 25G needle (Ex-PRESS entry system) was inserted into the $\mathrm{AC}$ through the center of the "blue line" at an angle parallel to the iris plane to create a path for the Ex-PRESS (model P-50) and then removed gently to avoid lateral movement that may extend the channel and cause aqueous humor to leak around the shunt. The Ex-PRESS shunt is preloaded on an injector. The shunt is introduced into the AC exclusively through the ostium created by the needle and released by applying pressure to the shaft of the inserter. The tip of the device was confirmed to be in the $\mathrm{AC}$ in the iris plane away from the cornea and without any iris obstruction.

The scleral flap is then sutured with interrupted 10-0 Nylon. Two to three sutures were typically required with the tightness adjusted depending on the resultant flow during inflation of the AC with balanced salt solution using a $27 \mathrm{G}$ needle through the temporal paracentesis to restrict flow to a "slow

\section{Results:}

One case showed migration and extrusion of the device four months postoperatively, which was excluded from the results (Figure 1).

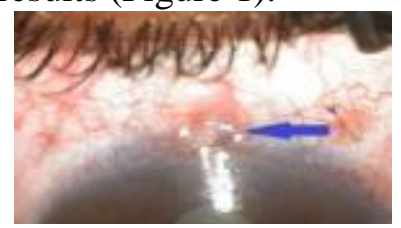

Figure 1: Impending extrusion of Ex-PRESS (blue arrow)

Patients included 3 males and 2 females. Studied patients' demographics and ocular characteristics preoperatively are summarized in tables 1 . The ocular characteristics one year postoperative are summarized in tables 2. Comparisons between pre- and post-operative data are summarized in tables 3 and 4 . There were 4 eyes $(80 \%)$ showed complete success (IOP $<21 \mathrm{mmHg}$ without treatment) and 1 eyes $(20 \%)$ showed qualified success (IOP $<21 \mathrm{mmHg}$ with anti-glaucoma medical treatment) 
Table 1: Preoperative demographics and ocular characteristics of studied patients

\begin{tabular}{|c|c|c|}
\hline Variable & Mean & $\begin{array}{c}\text { Std. } \\
\text { Deviation }\end{array}$ \\
\hline Age & 56.40 & 7.54 \\
\hline IOP & 35.76 & 9.04 \\
\hline BCVA & 1.65 & 0.47 \\
\hline NO. of Medications & 3.56 & 0.51 \\
\hline MD & -21.12 & 8.71 \\
\hline PSD & 6.70 & 2.66 \\
\hline RNFL & 56.80 & 17.49 \\
\hline GCC & 64.84 & 14.00 \\
\hline Endothelial CD & 2648.68 & 440.25 \\
\hline
\end{tabular}

Table 2: Postoperative ocular characteristics of studied patients

\begin{tabular}{|c|c|c|}
\hline Variable & Mean & $\begin{array}{c}\text { Std. } \\
\text { Deviation }\end{array}$ \\
\hline IOP after 1 month & 20.84 & 2.82 \\
\hline IOP after 3 months & 21.96 & 5.31 \\
\hline IOP after 6 months & 16.92 & 3.87 \\
\hline IOP after 1 year & 17.20 & 1.83 \\
\hline BCVA & 1.52 & 0.42 \\
\hline NO. of Medications & 0.92 & 1.08 \\
\hline Time to Re-enter Medications & 3.04 & 0.97 \\
\hline MD & -18.82 & 8.41 \\
\hline PSD & 6.12 & 2.76 \\
\hline RNFL & 62.80 & 18.91 \\
\hline GCC & 72.00 & 16.13 \\
\hline Endothelial Cell Density & 2804.72 & 482.14 \\
\hline
\end{tabular}

Table 3: Comparison of preoperative IOP and IOP 1, 3, 6 months and 1 year postoperatively

\begin{tabular}{|c|c|c|}
\hline & Postoperative IOP & $P$ value \\
\hline \multirow{3}{*}{$\begin{array}{c}\text { Preoperative IOP } \\
\text { (35.76 } \pm 9.04)\end{array}$} & 1 Month $(20.84 \pm 2.82)$ & \multirow{3}{*}{$<0.001$} \\
\cline { 2 - 2 } & 3 Months $(21.96 \pm 5.31)$ & \\
\cline { 2 - 2 } & 6 Months $(16.92 \pm 3.87)$ & \\
\cline { 2 - 2 } & 1 Year $(17.20 \pm 1.83)$ & \\
\hline
\end{tabular}

Table 4: Comparisons between data preoperatively and one year postoperative

\begin{tabular}{|c|c|c|c|}
\hline Variable & $\begin{array}{c}\text { Preoperative } \\
(\text { mean+sd) }\end{array}$ & $\begin{array}{c}\text { Postoperative } \\
(\text { mean+sd) }\end{array}$ & $P$ value \\
\hline BCVA & $1.65 \pm 0.47$ & $1.52 \pm 0.42$ & 0.002 \\
\hline NO. of Medications & $3.56 \pm 0.51$ & $0.92 \pm 1.08$ & $<0.001$ \\
\hline MD & $-21.12 \pm 8.71$ & $-18.82 \pm 8.41$ & \\
\hline PSD & $6.70 \pm 2.66$ & $6.12 \pm 2.76$ & 0.002 \\
\hline RNFL & $56.80 \pm 17.49$ & $62.80 \pm 18.91$ & \multirow{2}{*}{$<0.001$} \\
\hline GCC & $64.84 \pm 14.00$ & $72.00 \pm 16.13$ & \\
\hline Endothelial Cell Density & $2648.68 \pm 440.25$ & $2804.72 \pm 482.14$ & \\
\hline
\end{tabular}

\section{Discussion:}

Glaucoma is the leading cause of irreversible blindness worldwide and represents a significant public health concern. ${ }^{(7)}$ The Ex-Press GDD is a new method for standardizing trabeculectomy with outcomes quite similar to trabeculectomy reported in the literature. previous study by Takihara $^{(8)}$ showed that the rate of failure of trabeculectomy with MMC among patients with OAG was higher in pseudophakic eyes after phacoemulsification than in phakic eyes. Some studies ${ }^{(9-10)}$ indicated that patients with glaucoma who undergo cataract surgery exhibit resistance to maintaining target IOP after trabeculectomy. However, these studies included pseudophakic and aphakic eyes after extracapsular or intracapsular cataract extraction. Cataract extraction may cause extensive scarring in the superior location of the conjunctiva, where trabeculectomy should be performed. Phacoemulsification causes less 
Scar formation in the conjunctiva of patients with glaucoma.

In our study, the complete and qualified success rates were $80 \%$ and $20 \%$ respectively after 1 year of ExPRESS implantation. A recent retrospective study evaluated the surgical prognosis of trabeculectomy in 51 pseudophakic eyes after phacoemulsification among patients with OAG. ${ }^{(11)}$ In that study, the probability of success at 1 year after trabeculectomy in pseudophakic eyes was $78.6 \%$ for IOP of less than 21 $\mathrm{mmHg}$. The pathomechanism for failure of trabeculectomy in pseudophakic eyes is not fully understood. ${ }^{(12)}$

https://jamanetwork.com/journals/jamao phthalmology/fullarticle/ 1774027

eoi130184r15 Alterations in the nature of the AH may contribute to failure of trabeculectomy. Intraocular surgery causes ocular inflammation and breakdown of the blood-aqueous barrier, which may cause bleb failure after trabeculectomy. ${ }^{(13)}$ A high concentration of inflammatory cytokines has been shown in the $\mathrm{AH}$ of pseudophakic eyes with glaucoma. ${ }^{(14)}$

In our study, the number of antiglaucoma medications taken postoperatively $(0.92 \pm 1.08)$ was significantly lower than number of medications taken preoperatively $(3.56 \pm 0.51) \quad(P<0.001)$. In previous study by Liu et al, ${ }^{(15)}$ the number of glaucoma medications taken postoperatively was lower than the number of glaucoma medications taken pre-operatively $(0.94 \pm 0.96$ vs. $1.18 \pm$ 1.38). In a study comparing Ex-PRESS to trabeculectomy in an African origin population, ${ }^{(16)}$ the number of glaucoma medications used to control IOP postoperatively was reduced from $(3.82 \pm 0.8)$ at baseline to $(0.86 \pm 1.00)$ $(P=0.05)$ at 12 months for Ex-PRESS group.
VA may improve following surgery or may deteriorate following surgery either as a result of complications of surgery or progression of disease. Our results showed significant improvement of BCVA from $(1.65 \pm 0.47)$ LogMAR preoperatively to $\quad(1.52 \pm 0.42) \quad$ LogMAR postoperatively $(P=0.002)$. Good and Kahook $^{(17)}$ reported increased visual recovery following surgical intervention in patients undergoing ExPRESS surgery.

On the other hand, several investigators have noted a risk of central VA loss after filtration surgery in eyes with advanced glaucoma. ${ }^{(18-19)}$ Wagschal et $\mathrm{al}^{(20)}$ found that VA in patients treated with the Ex-PRESS implant did not differ significantly from baseline by 1 month postoperation $(P=0.17)$, and remained stable for all subsequent visits. In another prospective randomized trial by Beltran-Agullo et al, ${ }^{(21)}$ by month 1 , VA in the Ex-PRESS group was no longer significantly different from baseline $(P=0.23)$ and remained stable throughout 6 months of follow-up.

Regarding VF changes, our study showed improvement of global indices (MD \& PSD). MD improved from ($21.12 \pm 8.71)$ prepoeratively to ($18.82 \pm 8.41)$ one year postoperatively $(P<0.001)$. Also, PSD showed improvement from $(6.7 \pm 2.66)$ preoperatively to $(6.12 \pm 2.76)$ one year postoperatively $(P=0.002)$. However, GSS2, a parameter evaluate both MD and PSD simultaneously, showed no difference pre- and post-operatively $(P=0.327)$.

It has been shown previously ${ }^{(22-23)}$ that light sensitivity may increase diffusely across the VF when IOP is surgically reduced. In addition, local improvement in the VF was also found. ${ }^{(24)}$ However, improvement correlated positively, but nonsignificantly to the decrease in 
postoperative IOP: a larger reduction of IOP was associated with a higher number of improved clusters in the postoperative VF.

One study suggested that perimetric improvement after initiation of therapy occurs more often in younger individuals, ${ }^{(25)}$ a finding we could not confirm because no patient younger than 40 years of age was included in our study. It also has been reported that improvement occurs mostly in eyes with early VF loss, ${ }^{(26)}$ Hence the importance of control groups when studying treatment effects on a disease like glaucoma, when the outcome variable is derived from a psychophysical test whose results are known to be affected by perimetric learning effect. ${ }^{(27-28)}$

Regarding the OCT parameters, our results showed increase in thickness of both RNFL and GCC. RNFL thickness increased from $(56.80 \pm 17.49)$ um preoperatively to $(62.80 \pm 18.91)$ um one year postoperatively $(P<0.001)$. Moreover, GCC thickness increased from $(64.84 \pm 14.00)$ um preoperatively to $(72.00 \pm 16.73)$ um 1 year postopertively $(P<0.001)$. In a study by Sarkar, ${ }^{(29)}$ there was significant RNFL thickness changes found with significant reduction of IOP.

The physiologic basis of the improvement in RNFL thickness with IOP reduction is not clear. It has been suggested that IOP reduction results in less posterior bowing of the lamina cribrosa. ${ }^{(30-31)}$ The clinical implications of the RNFL thickness improvement are unclear, although previous studies have shown that there may be an associated improvement of visual function that corresponds to this improvement ${ }^{(32-33)}$ and similar findings have been reported by Lesk et al. ${ }^{(34)}$

Ali Aydin et al ${ }^{(35)}$ found a significant increase in overall mean peripapillary RNFL thickness after surgery as determined by OCT $(P<0.0001) .28$
(73.7\%) of 38 eyes had an IOP reduction $>30 \%$. The mean RNFL thickness increase $(0.5 \mathrm{um} / \mathrm{mmHg}$ decrease of IOP) was significantly correlated with the IOP reduction $(r=-$ $0.41 ; P=0.03)$. The possible explanation is that the increase of RNFL thickness may reflect the recovery of the compressed RNFL, which regains its original shape because of the IOP reduction. As compression on the axons is relieved with IOP reduction, the axons may recover their normal shape and size, with resultant increase in RNFL thickness.

In contrast, Chang et al ${ }^{(36)}$ studied 21 eyes of 21 glaucoma patients who underwent medical or surgical intervention to lower IOP. There was no significant change in the overall RNFL thickness associated with the lowering of IOP $(1.02 \pm 10.3$ $\mu \mathrm{m}, P=0.653)$. Quadrant analysis did not show any significant change in the RNFL thickness of any of the four quadrants. This might be due to intervention variation in his study.

As for the influence of Ex-PRESS mini glaucoma shunts on the cornea condition, our results revealed increase in endothelial $\mathrm{CD}$ from $(2648.68+440.25) \quad$ cells $/ \mathrm{mm}^{2}$ preoperatively to $(2804.73+482.14)$ cells $/ \mathrm{mm}^{2}$ one year postoperatively $(P<0.001)$.

In studies involving the use of animal models, it was noted that the material, of which the drainage device is made, affects the degree of cell loss. ${ }^{(37)}$ Thus, in comparison with silicone and polymethylmethacrylate (PMMA), phosphorycholine polymercoated PMMA (PC-PMMA) causes the least endothelial cells damage. There have been no reports in the literature showing the effect of stainless steel, of which the Ex-Press mini glaucoma shunt is made, on endothelial cells, whereas, in previous analysis, ${ }^{(38)}$ the 
CD loss \% is significantly higher than in the case of implantation of an AGV or Baerveldt glaucoma tube implant. ${ }^{(39)}$ In our study, there was an improvement in endothelial CD.

Such a difference between the findings may result from the fact that damage to endothelial cells after cataract surgery itself is documented. ${ }^{(40-41)}$ Furthermore, theories suggest that persistently elevated IOP directly or indirectly induces hypoxia, thereby damaging the endothelium. ${ }^{(42)}$ Thus IOP reduction decreases hypoxia and help improvement of CD. Moreover, Fiore et al. assume that the mechanism of endothelial damage may be associated with toxic effects of medications, preservatives contained in ophthalmic drops, and duration of treatment. ${ }^{(43)}$ Some researchers believe that patients taking three or four anti-glaucoma medications at the same time have lower CD compared to those taking only one or two preparations. ${ }^{(44)}$ So, reduction of postoperative medications used may explain the improvement in CD.

In this study, we can conclude that, ExPRESS is preferable for pseudphakic glaucoma. Previous studies reported that Ex-PRESS implantation is undoubtedly effective treatment methods for pseudphakic glaucoma, compared with the drug and laser therapy.

\section{References:}

1. van Oye $R$ and Gelisken $O$. Pseudophakic glaucoma. Int Ophthalmol. 1985;8(3):183-6.

2. Jamil AZ, Iqbal K, Ur Rahman F, et al. Effect of phacoemulsification on intraocular pressure. $\mathrm{J}$ Coll Physicians Surg Pak. 2011;21(6):34750.

3. Bhallil S, Andalloussi IB, Chraibi F, et al. Changes in intraocular pressure after clear corneal phacoemulsification in normal patients. Oman J Ophthalmol. 2009;2(3):111-3.

4. Călugăru M. Tratat clinic de glaucoma. 1998, Editura Dacia, ClujNapoca.

5. Minckler DS, Francis BA, Hodapp EA, et al. Aqueous shunts in glaucoma: a report by the American Academy of Ophthalmology. Ophthalmol. 2008;115(6):1089-98.

6. Francis BA, Singh K, Lin SC, et al. Novel glaucoma procedures: a report by the American Academy of Ophthalmology. Ophthalmol. 2011;118(7):1466-80.

7. Quigley HA and Broman AT. The number of people of glaucoma worldwide in 2010 and 2020. Br J Ophthalmol. 2006;90(3):262-7.

8. Takihara Y, InataniM, OgataIwao M, et al. Trabeculectomy for open-angle glaucoma in phakic eyes vs in pseudophakic eyes after phacoemulsification: A prospective clinical cohort study. JAMA Ophthalmol. 2014;132(1):69-76.

9. The Fluorouracil Filtering Surgery Study Group. Five year follow up of the fluorouracil filtering surgery study. Am J Ophthalmol. 1996;121(4):349-66.

10. Prata Júnior JA, Minckler DS, Baerveldt G, et al. Trabeculectomy in pseudophakic patients: postoperative 5-fluorouracil versus intraoperative mitomycin C antiproliferative therapy. Ophthalmic Surg. 1995;26(1):73-7.

11. Takihara $Y$, Inatani $M$, Seto $T$, et al. Trabeculectomy with mitomycin for open-angle glaucoma in phakic vs pseudophakic eyes after phacoemulsification. Arch Ophthalmol. 2011;129(2):152-7.

12. Broadway DC, Grierson I and Hitchings RA. Local effects of previous conjunctival incisional surgery and the subsequent outcome 
of filtration surgery. Am J

Ophthalmol. 1998;125(6):805-18.

13. Joseph JP, Grierson I and Hitchings RA. Chemotactic activity of aqueous humor: a cause of failure of trabeculectomies? Arch Ophthalmol. 1989;107(1):69-74.

14. Inoue T, Kawaji T, Inatani M, et al. Simultaneous increases in multiple proinflammatory cytokines in the aqueous humor in pseudophakic glaucomatous eyes. $\mathbf{J}$ Cataract Refract Surg. 2012;38(8):1389-97.

15. Liu JH, Lin HY, Tzeng SH, et al. Comparison of trabeculectomy with Ex-PRESS shunt implantation in primary-open-angle-glaucoma patients: A retrospective study. Taiwan J Ophthalmol. 2015;5(3):1203 .

16. Bustros YD, Fechtner $R$ and Khouri AS. Outcomes of Ex-PRESS and trabeculectomy in a glaucoma population of African origin: one year results. J Curr Glaucoma Pract. 2017;11(2):42-7.

17. Good TJ and Kahook MY. Assessment of bleb morphologic features and postoperative outcomes after Ex-PRESS drainage device implantation versus trabeculectomy. Am J Ophthalmol. 2011;151:50713.e1.

18. Costa VP, Smith M, Spaeth GL, et al. Loss of visual acuity after trabeculectomy. Ophthalmol. 1993;100:599-612.

19. Levene RZ. Central visual field, visual acuity, and sudden visual loss after glaucoma surgery. Ophthalmic Surg. 1992;23:388-94.

20. Wagschal LD, Trope GE, Jinapriya D, et al. Prospective randomized study comparing ExPRESS to trabeculectomy: 1-year results. J Glaucoma. 2015;24(8):6249.

21. Beltran-Agullo L, Trope GE, Jin Y, et al. Comparison of visual recovery following Ex-PRESS versus trabeculec $\neg$ tomy: results of a prospective randomized controlled trial. J Glaucoma. 2015;24:181-6.

22. Boissonot M, Hirsch AL, Rovira JC, et al. Evolution du champ visuel enperimetrie automatisee dans le glaucoma chronique equilibrepar trabeculectomie. J Fr Ophtalmol. 1992;15:645-50.

23. Gandolfi S. Improvement of visual field indices after surgical reduction of intraocular pressure. Ophthalmic Surg. 1995;26:121-6.

24. Vuori ML, Vainio-Jylhä $E$ and Viitanen TT. Localised changes in glaucomatous visual fields after trabeculectomy. Acta Ophthalmol. Scand. 2001;79:468-71.

25. Flammer J and Drance SM. Effect if acetazolamide on the differential threshold. Arch Ophthalmol. 1983;101:1378-80.

26. Wright TM, Goharian I, Gardiner SK, et al. Short-term enhancement of visual field sensitivity in glaucomatous eye following surgical intraocular pressure reduction. Am J Ophthalmol. 2015;159:378-85.

27. Wild JM, Searle AE, DenglerHarles M, et al. Long-term followup of baseline learning and fatigue effects in the automated perimeter of glaucoma and ocular hypertensive patients. Acta Ophthalmol. 1991;69:210-6.

28. Heijl A and Bengtsson B. The effect of perimetric experience in patients with glaucoma. Arch Ophthalmol. 1996;114:19-22.

29. Sarkar KC, Das P, Pal R, et al. Optical coherence tomographic assessment of retinal nerve fiber layer thickness changes before and after glaucoma filtration surgery. Oman J Ophthalmol. 2014;7(1):3-8.

30. Quigley HA. The pathogenesis of reversible cupping in congenital 
glaucoma. Am J Ophthalmol. 1977;84:358-70.

31. Albon J, Purslow PP, Karwatowski WS, et al. Age related compliance of the lamina cribrosa in human eyes. $\mathrm{Br} \mathrm{J}$ Ophthalmol. 2000;84:318-23.

32. Yildirim E, Bilge AH and Ilker $\mathrm{S}$. Improvement of visual field following trabeculectomy for open angle glaucoma. Eye. 1990;4:103-6.

33. Tsai CS, Shin DH, Wan JY, et al. Visual field global indices in patients with reversal of glaucomatous cupping after intraocular pressure reduction. Ophthalmol. 1991;98:1412-9.

34. Lesk MR, Spaeth GL, AzuaraBlanco A, et al. Reversal of optic disc cupping after glaucoma surgery analyzed with a scanning laser tomograph. Ophthalmol. 1999;106:1013-8.

35. Aydin A, Wollstein G, Price LL, et al. Optical coherence tomography assessment of retinal nerve fiber layer thickness changes after glaucoma surgery. Ophthalmol. 2003;110:150611.

36. Chang PT, Sekhon N, Budenz DL, et al. Effect of lowering intraocular pressure on optical coherence tomography measurement of peripapillary retinal nerve fiber layer thickness. Ophthalmol. 2007; 114:2252-8.

37. Lim KS. Corneal endothelial cell damage from glaucoma drainage device materials. Cornea. 2003;22(4):352-4.

38. Konopinska J, Deniziak M, Saeed E, et al. Prospective randomized study comparing combined phacoExPress and phacotrabeculectomy in open angle glaucoma treatment: 12month follow-up. J Ophthalmol. 2015;2015:720109.

39. Saheb H, Gedde SJ, Schiffman JC, et al. Outcomes of glaucoma reoperations in the Tube Versus Trabeculectomy (TVT) study. Am J Ophthalmol. 2014;157(6):117989.e2.

40. Park JH, Lee SM, Kwon JW, et al. Ultrasound energy in phacoemulsification: a comparative analysis of phaco-chop and stop-andchop techniques according to the degree of nuclear density. Ophthalmic Surg Lasers Imaging. 2010;41(2):236-41.

41. Moschos MM, Chatziralli IP and Sergentanis TN. Viscoat versus Visthesia during phacoemulsification cataract surgery: corneal and foveal changes. BMC Ophthalmol. 2011;11(1):9.

42. Ollivier FJ, Brooks DE, Komaromy AM, et al. Corneal thickness and endothelial cell density measured by non-contact specular microscopy and pachymetry in Rhesus macaques (Macaca mulatta) with laser-induced ocular hypertension. Exp Eye Res. 2003;76(6):671-7.

43. Fiore PM, Richter G, Arzeno CU, et al. The effect of anterior chamber depth on endothelial cell count after filtration surgery," Arch Ophthalmol. 1989;107(11):1609-11.

44. Lass JH, Khosrof SA, Laurence JK, et al. A double-masked, randomized, 1-year study comparing the corneal effects of dorzolamide, timolol, and betaxolol. Arch Ophthalmol. 1998;116(8):1003-10. 